\title{
Successful outcome of placenta previa percreta with bladder invasion
}

\author{
Syeda Sayeeda, Nahreen Akhter, Firoza Begum and Sajid Hasan
}

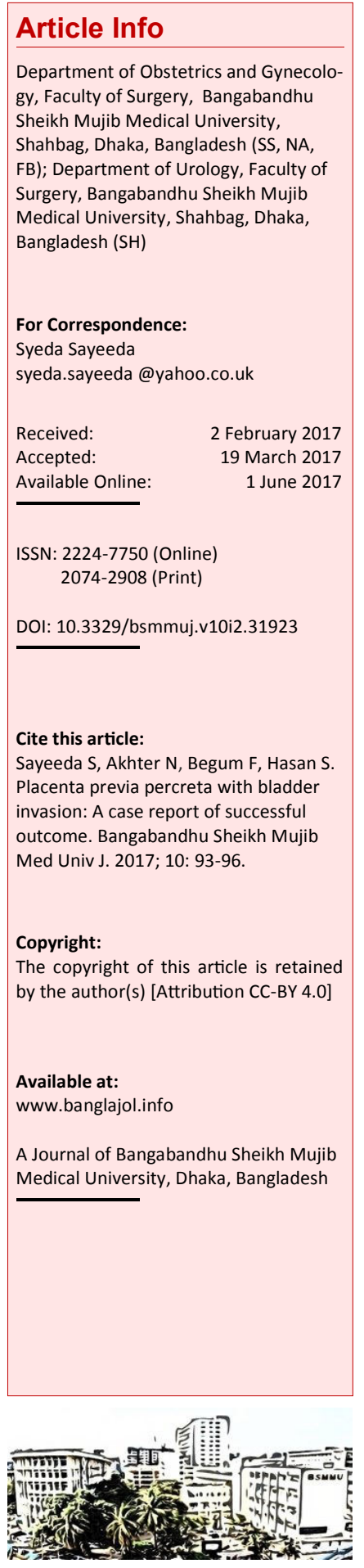

\section{Abstract}

A 41 year old multiparous lady, with previous history of one cesarean section presented at her 24 weeks of gestation with frank hematuria. The case was diagnosed as placenta previa percreta with the bladder involvement by ultrasound doppler and confirmed by MR urogram. So, peripartum hysterectomy was planned. On opening of the abdomen, a hugely distended bladder was found, which when retracted engorged blood vessels were found over the lower segment of uterus. Baby was delivered by giving a transverse incision in the upper segment. By keeping placenta in situ, total abdominal hysterectomy was done with quick successive clamping. Severe per-operative bleeding was occurred. Bladder irrigation started following total abdominal hysterectomy. Continuous small clots were coming out through catheter. A large old blood clot was removed by cystostomy done by an urologist. A sprouting vessel and a linear injury were noticed at the base of the bladder. The vessel was ligated and the injury was repaired. After proper hemostasis, the abdomen was closed in layers. The patient was shifted to ICU. Patient developed complications like MI, watery diarrhoea, low grade fever which was managed accordingly. She was discharged healthy on her 19th post-operative day.

\section{Introduction}

Placenta percreta is a rare condition which might be complicated by attachment of the placenta to surrounding structures or organs, such as urinary bladder or rectum. To manage such a case requires multidisciplinary approach because of the risk of massive hemorrhage, bladder damage, and the development of disseminated intravascular coagulation. It is a potentially fatal condition, and the mortality rate is correlated to the extent of involvement of surrounding structures. Mortality rate has been estimated to be as high as 9.5 and $24 \%$ for mother and child, respectively, when placenta percreta is complicated by bladder invasion. $\underline{1}$ Herein, a case of placenta previa percreta with the bladder involvement, diagnosed and confirmed by ultrasound doppler and MR urogram, was reported to highlight the catastrophic nature of this clinical entity, which if managed appropriately was associated with a better outcome.

\section{Case Report}

A 41 year old multiparous lady (p-2+1), with previous history of one cesarean section presented at her 24 weeks of gestation with frank hematuria for 11 days. She had mild per vaginal bleeding and repeated urinary tract infection in her early pregnancy. She suddenly developed acute retention of urine at her 22 weeks of gestation, which was relieved by Foley's catheter keeping in situ for 48 hours and receiving antibiotic in her locality. Subsequent to removal of the catheter, she developed frank hematuria and got herself admitted in the Department of Urology, Dhaka Medical College Hospital for further management. Several investigations, including complete blood count, ultrasonogram of pregnancy profile with doppler study, cystoscopy and MR urogram of the pelvic organs were done and injection ceftriaxon, tranexamic acid, continuous bladder irrigation and two units of blood transfusion were given. Ultrasonography revealed complete placenta percreta with likely invasion of the bladder wall, as evidenced by mildly thickened posterior wall and increase vascularrity. Cystoscopy did not reveal the bladder invasion, only features of cystitis and moderate amount of blood clot were found. MR urogram revealed the placenta percreta with full thickness myometrial invasion, with adjacent thickened urinary bladder wall suggesting minimal focal invasion. With the above management, hematuria continued. So, the patient moved on for further opinion and got herself admitted into the Fetomternal Medicine Unit of the Department of Obstetrics and Gynecology, Bangabandhu Sheikh Mujib Medical 
University. All the investigations that were done were reviewed clinically and the case was confirmed as placenta previa percreta with bladder involvement. Several investigations were done and found her severely anemic (hemoglobin- $6.2 \mathrm{gm} /$ $\mathrm{dL}$ ), diabetic (uncontrolled), thyroid status, liver function test, kidney function test and coagulation profile normal. Insulin was started and four units of whole blood was transfused. The patient was made aware of the potential obstetric complications. Peripartum hysterectomy was planned in collaboration with a multidisciplinary team including obstetricians, urologist and anesthesiologist. On opening of the abdomen, a hugely distended bladder was found (Figure 1), which when retracted large vascular channels were visible at the level of the lower segment of uterus. In order to avoid placental bed a transverse incision was made in the upper segment and a stillborn baby was delivered. By keeping placenta in situ, the total abdominal hysterectomy was done with quick successive clamping (Figure 1). Severe peroperative bleeding occurred as placenta become partially separated during the procedure and was secured. Bladder irrigation started following total abdominal hysterectomy. Several small clots were coming out continuously through the catheter. So, a cystotomy was created by urologist to facilitate removal of

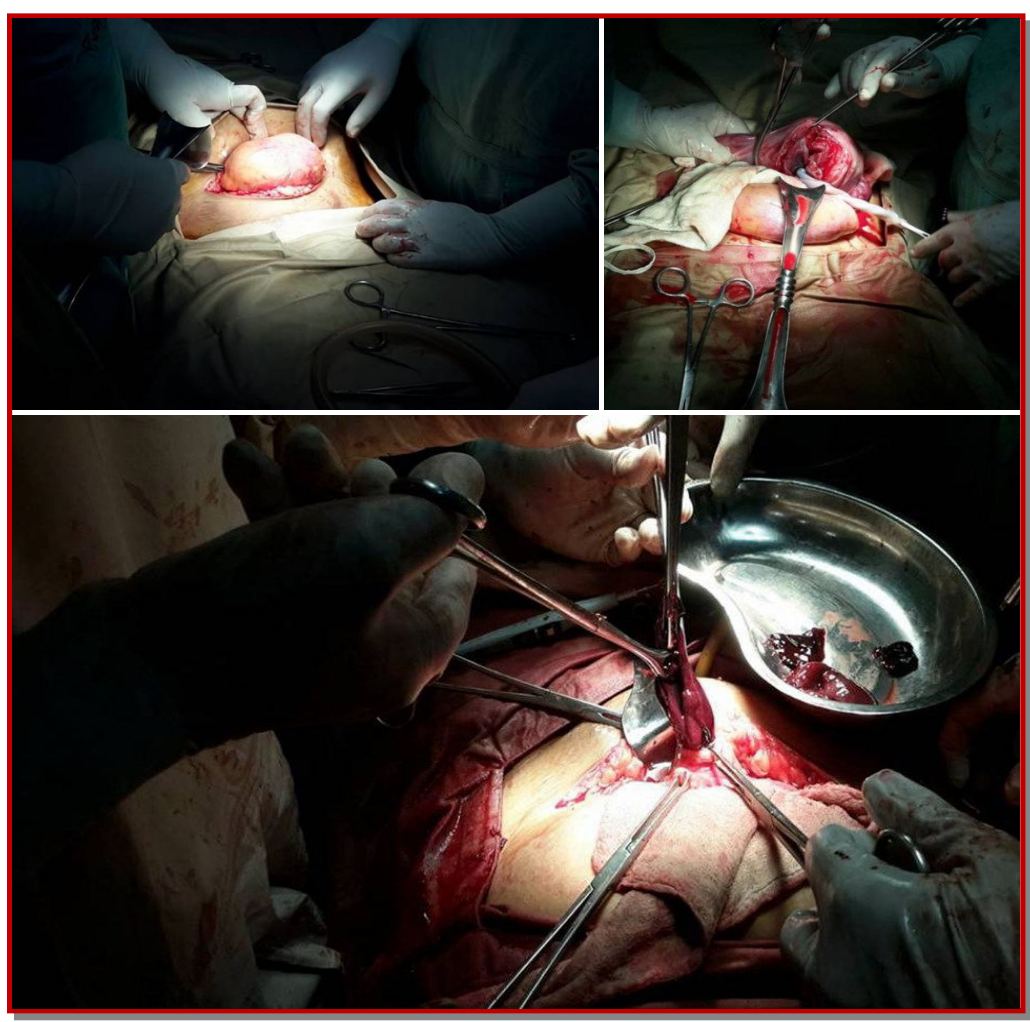

Figure 1: On opening the abdomen, a hugely distended bladder popped up (Upper left); Hysterectomy was going on keeping placenta in situ (Upper right); A large blood clot along with several small pieces of clots were removed through cystotomy wound (Lower) blood clots (Figure 1). A sprouting vessel and a linear injury were noticed at the base of the bladder. The vessel was ligated and the injury was repaired (by $2 / 0$ vicryl) followed by repair of cystotomy wound in two layers. After proper hemostasis and keeping a drain tube in situ, abdomen was closed in layers. The patient was shifted to the intensive care unit and was cared for in ICU for seven days. Patient's postoperative course was complicated by MI, watery diarrhea, electrolyte imbalance and low grade fever which was managed accordingly. She also developed moderate size pelvic hematoma (diagnosed by ultrasonography) and was managed conservatively. Total 16 units of blood were transfused during pre-, per- and post-operative period. The catheter was removed on her 16th postoperative day and she was discharged healthy on 19th postoperative day

\section{Discussion}

The incidence of placenta percreta has risen substantially due to the increased rate of cesarean sections. A 50-fold rise has been reported during the past 50 years, to a currently estimated 1 in 1000 pregnancies. The incidence of concomitant bladder invasion is much lower, occurring in approximately 1 in 10,000 births. 2 According to ACOG committee opinion, the incidence rate of PP varies between $1 / 210$ to $1 / 2500$ births. 3

Major risk factors for placenta percreta include advanced maternal age, multiparity, placenta previa, and prior uterine scarring. 4 Since the rate of cesarean section has been increasing, so has the incidence of placenta previa percreta. Clark and colleagues, $\underline{5} 30$ have shown that the risk of placenta previa increases proportionately with the number of prior cesarean deliveries $(0.26 \%$ in an unscarred uterus, and up to $10 \%$ in woman with history of at least four prior cesarean section). Again, they have shown that the association of placenta previa and prior uterine scarring greatly increases the chance to develop a placenta accreta (5\% risk in an unscarred uterus, to $67 \%$ risk in women with four prior cesarean sections). In another study 6 authors found that, in the presence of a placenta previa, the risk of placenta accreta was 3,11, 40, 61 and $67 \%$ for the first, second, third, fourth, and fifth or greater repeat cesarean deliveries, respectively. Like most others reported in their studies,,, 7 the patient in this case was a 41 year old multiparous lady with complete placenta previa percreta with history of one prior cesarean section. ${ }^{8}$

Placenta percreta during pregnancy, may be asymptomatic, or may present with mild to severe antepartum hemorrhage or lower abdominal pain ${ }^{8-}$ $\underline{10}$ or gross hematuria when bladder is invaded. 10 The patient, in this case of placenta previa percreta 
with bladder involvement, presented with frank hematuria which might be due to erosion of placental vessels. She also had mild per vaginal bleeding and repeated urinary tract infection in her early pregnancy.

The diagnosis of placenta percreta might be made during prenatal screening ultrasound. However, bladder involvement is usually not identified until the time of delivery. Symptoms such as gross hematuria, which might be expected, occur in only approximately in $25 \%$ of cases. 11 Hence, a high index of suspicion is needed in any pregnant patient presenting with gross hematuria. In this case, sonography done at her 23 weeks of gestation revealed plecenta previa type IV, with most likely invasion of bladder wall as evidenced by mildly thick posterior wall and increase vascularity. This finding was confirmed by MRI. But cystoscopy could not reveal placental tissue invasion other than features of cystitis and moderate amount of blood clot. Canonico et al $\underline{10}$ and others also found role of sonography, MRI and cystoscopy in diagnosing and confirmation of placenta percreta with bladder invasion same as in this case.

Diagnosis of placenta accreta during antepartum clearly aids in the approach and management of the disorder. It also helps to anticipate and recognize complications earlier. $\underline{4}$ Herein, following diagnosis of placenta previa percreta antenatally, hysterectomy was planned by keeping placenta in situ, as seen in other cases. $\frac{8}{\text { Th }}$ The most influential variable on maternal outcome is not attempting to remove the placenta. A retrospective study by Yap et al $\underline{12}$ showed that placental removal before hysterectomy resulted in increased maternal morbidity. A recent review also advised against attempts at placental removal before hysterictomy. $\underline{13}$

On opening the abdomen, urinary bladder was found hugely distended which might be due to accumulated blood clot and urinary retention. Avoiding the large vascular channels visible at the level of the lower uterine segment, a transverse incision was made in the upper segment to deliver the baby. Subsequently, total abdominal hysterictomy was done with quick successive clamping by keeping placenta in situ. But, as the placenta become partially separated during the procedure, huge bleeding occurred and needed 4 units of whole blood transfusion during the procedure and 8 units following the procedure. The average blood loss at delivery in women with placenta accreta is 3,000-5,000 mL. . 4 Smith et al 7 described that their patient required resuscitation with 37 units of various blood products in total during the procedure of total abdominal hysterectomy and adhesiolysis. Silvia et al $\underline{4}$ showed in their report, they needed $1050 \mathrm{~mL}$ of allogenic RBC and 1,400 $\mathrm{mL}$ of FFP following manual removal of placenta.
Major and minor complications of placeta accreta include: Massive hemorrhage, leading to disseminated intravascular coagulopathy; the need for hysterectomy; uterine wall rupture, uterine inversion secondary to attempted manual removal of placenta, retained product of conception, injury to the ureters, bladder, bowel, or neurovascular structures; fistula formation, adult respiratory distress syndrome; acute transfusion reaction; electrolyte imbalance; and renal failure. $.4,8$ In this case, the patient bleeded profusely during hysterectomy. In total, 16 units of blood were required during ante, intra and postpartum period for its resuscitation. The patient developed MI, electrolyte imbalance, fever and watery diarrhea during her postpartum period as a consequence of prolonged operative procedure, massive hemorrhage, huge blood transfusion and administration of several antibiotics, but subsequent recovery was uneventful.

\section{Conclusion}

A high index of suspicion for placenta percreta with bladder invasion is required when evaluating pregnant woman with a history of cesarean delivery and placenta previa who present with hematuria and lower urinary tract symptoms. Ultrasonogram of pregnancy profile and MRI of pelvic region may assist in establishing the diagnosis preoperatively. With proper planning and multidisciplinary approach maternal morbidity and mortality can be reduced.

\section{References}

1. Price FV, Resnik E, Heller KA, Christopherson WA. Placenta previa percreta involving the urinary bladder: A report of two cases and review of the literature. Obstet Gynecol. 1991; 78: 508-11.

2. Abbas F, Talati J, Wasti S, Akram S, Ghaffar S, Qureshi R. Placenta percreta with bladder invasion as a cause of life threatening hemorrhage. J Urol. 2000; 164: 1270-74.

3. ACOG Committee on Obstetric Practice. ACOG committee opinion. Number 266, January, 2002: Placenta accreta. Obstet Gynecol. 2002; 99: 169-70.

4. Silver LE, Hobel CJ, Lagasse L, Luttrull JW, Platt LD. Placenta previa percreta with bladder involvement: New considerations and review of the literature. Ultrasound Obstet Gynecol. 1997; 9: 131-38.

5. Clark SL, Kooninas PP, Phelan JP. Placenta previa/ accrete and non-cesarean section. Obstet Gynaecol. 1975; 66: 89-92.

6. Silver RM, Landon $\mathrm{MB}$, Rouse $\mathrm{DJ}$, Leveno $\mathrm{KJ}$, Spong CY, Thom EA, Moawad AH, Caritis SN, Harper M, Wapner RJ, Sorokin Y, Miodovnik M, 
Carpenter M, Peaceman AM, O'Sullivan MJ, Sibai B, Langer O, Thorp JM, Ramin SM, Mercer BM. Maternal morbidity associated with multiple repeat cesarean deliveries. National Institute of Child Health and Human Development Maternal-Fetal Medicine Units Network. Obstet Gynecol. 2006; 107: 1226-32.

7. Smith ZL, Shegal SS, Arsdalen KNV, Goldstein IS. Placenta percreta with invasion into the urinary bladder. Urol Case Rep. 2014; 2: 31-32.

8. Sulatana N, Mohyuddin S, Riaz M, Niaz WA, Murtaza B. Management of placenta percreta with bladder invasion. J Pioneer Med Sci. 2015; 5: 31-33.

9. Faranesh R, Romano S, Shalev E, Salim R. Suggested approach for management of placenta percreta invading the urinary bladder. Obstet Gynecol. 2007; 110: 512-15.

10. Canonico S, Arduini M, Epicoco G, Luzi G, Arena
S, Clerici G, Affronti G. Placenta previa percreta: A case report of successful management via conservative surgery. Case Rep Obstet Gynecol. 2013; 2013: 1-3.

11. Takai N, Eto M, Sato F, Mimata H, Miyakawa I. Placenta percreta invading the urinary bladder. Arch Gynecol Obstet. 2005; 271: 274-75.

12. Yap YY, Pervin LC, Pain SR, Wong SF, Chan Y. Manual removal of suspected placenta accreta at caesarean hysterectomy. Int J Gynecol Obstet. 2008; 100: 186-87.

13. Oyelese Y, Smulian JC. Placenta previa placenta accreta and vasa previa. Obstet Gynecol. 2006; 107: 927-41.

14. Hudon L, Belfort MA, Broome DR. Diagnosis and management of placenta percreta: A review. Obstet Gynecol Surg. 1998; 53: 509-17. 